\title{
Alignment of the ATLAS inner detector tracking system
}

\author{
Heather M. Gray ${ }^{1}$ \\ California Institute of Technology, \\ 1200 East California Boulevard, Pasadena, CA 91125, U.S.A. \\ Columbia University, \\ 538 W 120th Street, New York, NY 10027, U.S.A. \\ E-mail: heather.gray@cern.ch
}

ABSTRACT: ATLAS is a general-purpose experiment designed to study the high-energy collisions produced by the Large Hadron Collider. It is equipped with a sophisticated tracking system, which will reconstruct the tracks of charged particles and determine their production and decay vertices. Accurate alignment of this tracking system is necessary for ATLAS to achieve its physics goals. The methods used to achieve alignment are discussed and results from both Monte Carlo studies and cosmic data are presented.

KEYWORDS: Particle tracking detectors; Solid state detectors; Detector alignment and calibration methods (lasers, sources, particle-beams)

\footnotetext{
${ }^{1}$ Corresponding author. On behalf of the ATLAS Collaboration
} 


\section{Contents}

1 Introduction 1

2 Track-based alignment 2

2.1 Global $\chi^{2}$ alignment algorithm 3

2.2 Local $\chi^{2}$ alignment algorithm 4

2.3 Robust Alignment algorithm 4

2.4 Weak modes 4

3 Alignment procedure $\quad 4$

4 Software tests of alignment algorithms 5

5 Results from cosmic rays 5

6 Frequency scanning interferometry 6

$\begin{array}{lll}7 & \text { Conclusion } & 7\end{array}$

\section{Introduction}

ATLAS [1, 2] is one of two multi-purpose detectors built to probe the collisions produced by the Large Hadron Collider (LHC). The LHC will collide protons with a centre-of-mass energy of $14 \mathrm{TeV}$ with first collisions expected in 2009. Once the LHC reaches its design luminosity of $10^{34}$ $\mathrm{cm}^{-2} \mathrm{~s}^{-1}, 1000$ charged tracks are expected on average in each event.

The ATLAS Inner Detector [3, 4] comprises three sub-detectors, which use complementary technologies to obtain optimal track reconstruction in the high-multiplicity LHC environment. The silicon pixel detector $[5,6]$ lies closest to the interaction point consists of 1744 modules and has an intrinsic analog measurement accuracy of $10 \mu \mathrm{m}(\mathrm{R}-\phi) \times 115 \mu \mathrm{m}(z)$ in the barrel. The SemiConductor Tracker (SCT) is a silicon strip detector consisting of 4088 modules with an intrinsic barrel measurement accuracy of $17 \mu \mathrm{m}(R-\phi) \times 589 \mu \mathrm{m}(z)$. The Transition Radiation Tracker (TRT) uses 176 modules containing gas-filled straw tubes with an intrinsic accuracy of $130 \mu \mathrm{m}$. Each sub-detector has a barrel centered on the interaction point and two end-caps on either side (figure 1).

Alignment of the Inner Detector is crucial to obtain the required tracking performance. The alignment procedure aims to determine the positions of the modules of each sub-detector. Treating these as rigid bodies means that each silicon module has six degrees of freedom. To obtain track parameter uncertainties within $20 \%$ of the intrinsic resolution [1], silicon module positions must to be known to an accuracy of $10 \mu \mathrm{m}$ in the most sensitive coordinate. ATLAS will follow a number 


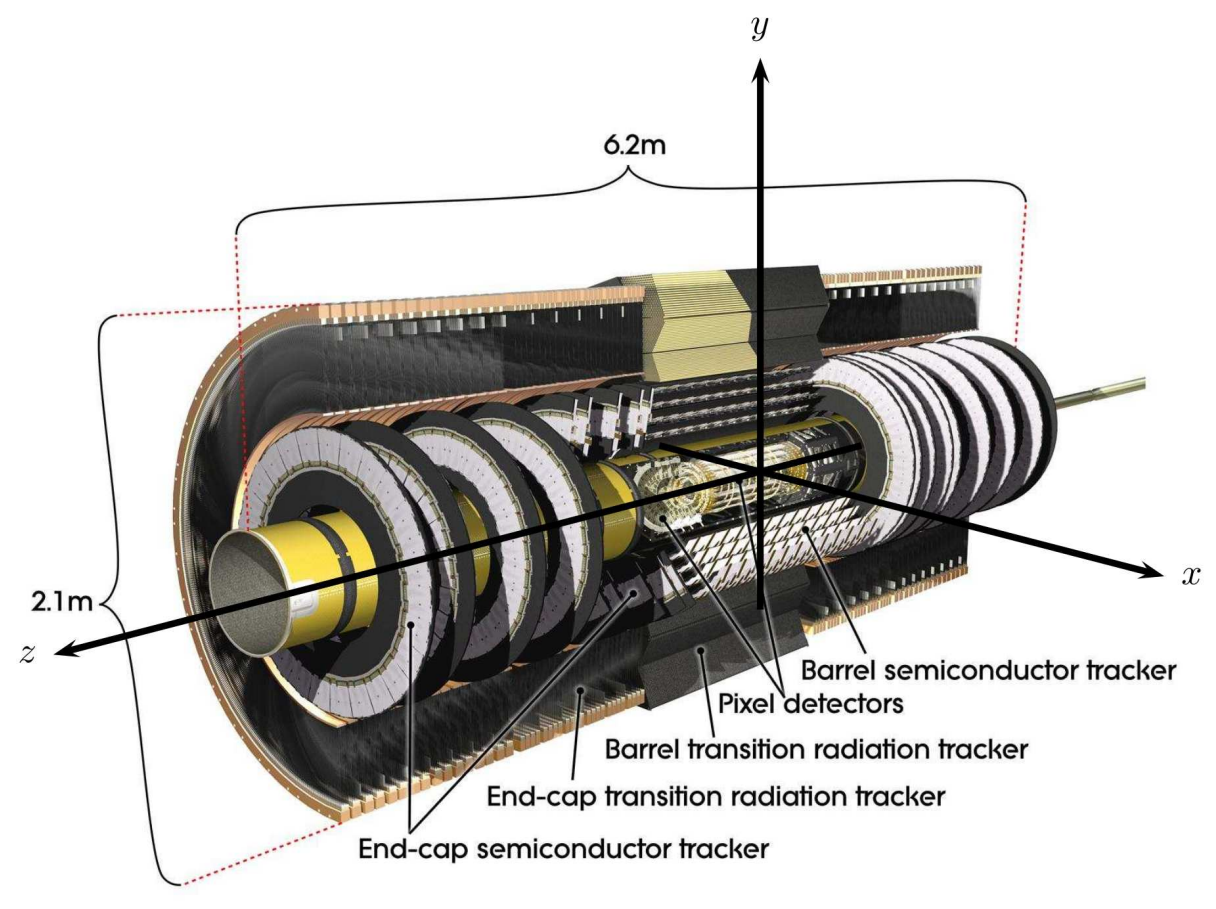

Figure 1. View of the ATLAS Inner Detector

of complementary strategies to obtain these goals. The build precision of the silicon modules is approximately $100 \mu \mathrm{m}$.

Alignment constants will be calculated using $0\left(10^{6}\right)$ tracks from collisions, but cosmic data has been used to obtain preliminary results. Optimal algorithm performance is obtained by combining information from data-sets sensitive to different types of misalignments, including cosmic rays and beam halo, with information from tracks from collisions.

During data-taking, a dedicated stream of isolated high $p_{T}$ tracks will be used to derive alignment constants. Alignment quality will be monitored using both basic distributions (including residuals and hit efficiencies) and certain physics observables (such as resonance masses and $\mathrm{E} / \mathrm{p}$ ). If the alignment monitor indicates degradation, alignment corrections will be recomputed and updated within 24 hours. This paper will focus on the alignment of the silicon detectors and present recent results obtained from cosmic data-taking with the full inner detector after installation underground.

The co-ordinate system used in ATLAS is a right-handed co-ordinate system, with the z-axis parallel to the beam axis and the $\mathrm{x}$-axis pointing to the centre of the LHC (see figure 1). Rotations around the axes are denoted as $\alpha, \beta$ and $\gamma$ for the $\mathrm{x}-, \mathrm{y}$ - and $\mathrm{z}$-axes respectively.

\section{Track-based alignment}

There are six degrees of freedom per module (three of translation, three of rotation), neglecting module deformations such as bending and twisting, which have not been considered yet. This means that the silicon modules alone have almost 35000 degrees of freedom. These cannot be 
measured directly, but must be inferred from the fit quality of reconstructed tracks. This is done by minimising a $\chi^{2}$ function of track-hit residuals. The residual for each individual track is defined as the distance between the reconstructed hit and the location predicted by the track fit.

The $\chi^{2}$ function is defined as follows:

$$
\chi^{2}=\sum_{\text {hits }} r^{T} V^{-1} r
$$

with

- $r$ : distance between hits and fitted tracks (vector of track residuals)

- $V$ : covariance matrix of the hit residuals

The sum over hits should be taken to represent the sum over hits on tracks for the events in the datasets. The general solution to minimise this $\chi^{2}$ is:

$$
\delta a=-\left(\sum_{\text {hits }} \frac{d r^{T}}{d a} V^{-1} \frac{d r}{d a}\right)^{-1}\left(\sum_{\text {hits }} \frac{d r^{T}}{d a} V^{-1} r\right)
$$

with

- $a$ : alignment parameters

- $\delta a$ : alignment corrections

The large number of degrees of freedom, mean that minimising eq. (2.1) involves solving systems of equations with tens of thousands of parameters. Therefore, multiple approaches have been pursued. Each uses different levels of approximation and can be iterated to improve precision.

\subsection{Global $\chi^{2}$ alignment algorithm}

The Global $\chi^{2}$ algorithm [7] attempts to solve eq. (2.2) while including all correlations between the different modules. This means that the derivative can be expanded as:

$$
\frac{d r}{d a}=\frac{\partial r}{\partial a}+\frac{\partial r}{\partial \pi} \frac{d \pi}{d a}
$$

with

- $\pi$ : track paremeters; typically $q / p, d_{0}, z_{0}, \phi_{0}$ and $\theta_{0}$

Solving eq. (2.2) is a significant numerical challenge because it requires inverting a symmetric matrix of degree 35000 . Special care must be taken because the system is under-constrained, making the matrix inherently singular. It can be solved either by diagonalisation or using sparse matrix techniques. Sparse matrix techniques require less storage space and are less time consuming but are less reliable than the direct solution. They also require preconditioning by adding damping terms, with the magnitude of the expected misalignment, to each degree of freedom. 


\subsection{Local $\chi^{2}$ alignment algorithm}

The Local $\chi^{2}[8,9]$ algorithm also uses eq. (2.2), but neglects correlations between different detector elements by replacing the full derivative (eq. (2.3)) by the partial derivative: $\frac{d r}{d a} \rightarrow \frac{\partial r}{\partial a}$. In this way, the alignment matrix is reduced to a series of $6 \times 6$ matrices, which can be inverted quickly, in contrast to the large matrix of the Global $\chi^{2}$ method. The alignment corrections are then computed with

$$
\delta a_{i}=-\left(\sum_{\text {hits }} \frac{\partial r_{i}^{T}}{\partial a} \frac{1}{\sigma_{i}^{2}} \frac{\partial r_{i}}{\partial a}\right)^{-1}\left(\sum_{\text {hits }} \frac{1}{\sigma_{i}^{2}} \frac{\partial r_{i}^{T}}{\partial a} r_{i}\right)
$$

where $\sigma_{i}$ is the standard deviation of the $i$-th residual. Correlations between modules are restored by computing the alignment constants iteratively, updating the geometry of the detector and then re-fitting the tracks.

\subsection{Robust Alignment algorithm}

The Robust Alignment (RA) algorithm [10-12] uses physical overlaps between modules to improve residual calculation. It is an iterative method, and within each iteration, alignment corrections are calculated using mean and overlap residuals. The overlap residuals relate the module position to that of its neighbours using overlaps in the $\phi$ and $z$ directions. The current implementation of the RA algorithm aligns only the in-plane translational degrees of freedom.

\subsection{Weak modes}

Minimisation of track residuals is necessary but not sufficient to align the Inner Detector. Certain global distortions preserve the helical trajectory of the track and leave the $\chi^{2}$ almost unchanged, but, nevertheless, lead to biases on track parameters. One of these is the so-called telescoping mode, which is when different layers are displaced in $\mathrm{z}$ with the displacement proportional to the radial distance from the interaction point.

The weak mode spectrum depends on the data-set used to produce the alignment constants and can therefore be constrained and eliminated by using tracks with different topologies. These include tracks from the interaction point with the primary vertex as an additional constraint; cosmic rays tracks, which provide tracks displaced in $\mathrm{z}$ and tracks connecting the upper and lower half of the detector; tracks from the beam halo to constrain the alignment of the end-cap regions; and tracks passing through the overlapping regions of modules.

\section{Alignment procedure}

The alignment procedure is broken down into several steps, referred to as levels. In the first step, or Level 1, the gross detector structures (the barrel and end-caps of the sub-detectors) are aligned with respect to each other. By considering these as rigid bodies with only six degrees of freedom, fewer tracks are needed to obtain alignment parameters. Secondly, for Level 2, the individual barrel layers and end-cap disks are aligned with respect to each other and, finally, Level 3, each individual module. Alignment results for various intermediate structures are additionally being calculated. For the pixel detector, for example, these include alignment results for the half shells of the barrel and results for the structural units of the barrel, staves of 13 modules, and the end caps, sectors of 6 modules. 


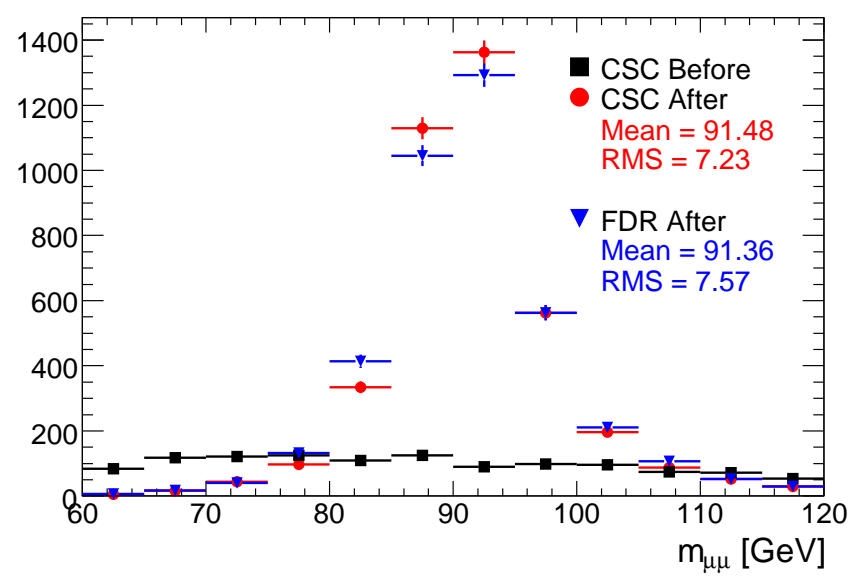

Figure 2. Invariant mass of muon pairs from $Z \rightarrow \mu \mu$ decays. Results using Si-tracks are shown for the CSC before alignment (black squares); the CSC after alignment (red circles) and the FDR after calculating the alignment constants(blue triangles).

\section{Software tests of alignment algorithms}

For the Computing System Commissioning (CSC) exercise, events were simulated with a misaligned inner detector geometry, but reconstructed with the nominal geometry. This studied the performance of the different alignment approaches within a large-scale scenario similar to what might be expected in first data. The misalignments input to the simulation were $\mathrm{O}(1 \mathrm{~mm})$ between sub-detectors and $\mathrm{O}(100 \mu \mathrm{m})$ between modules. Samples containing approximately 1 million muon tracks and events containing cosmic rays were used to calculate the alignment constants. Figure 2 shows the invariant mass of muon pairs from $Z \rightarrow \mu \mu$ decays using tracks reconstructed with hits in the silicon detectors only. In the CSC sample before alignment (black squares) the peak is not visible, but after a first-pass alignment (red squares) it is clearly visible with a width of $7 \mathrm{GeV}$.

The Final Dress Rehearsal (FDR) was the final software test before detector commissioning. It was a real-time test of the readiness of the software and computing infrastructure to handle LHC data. Alignment algorithms were run to produce alignment constants on the 24-hours time scale expected to be maintained during data-taking. The algorithm run began by determining the beam spot, aligning the silicon detectors, then the TRT, determining the origin using the global centre of gravity of tracks and finally rerunning the beam spot determination. An external constraint was applied by assuming the centre of gravity to be fixed while running the alignment algorithms. Alignment results were consistent with those obtained in the CSC (see figure 2 and also figure 3) and validated the readiness of the alignment infrastructure for first data.

\section{Results from cosmic rays}

Before collision data is available, cosmic rays are used to perform a first-pass of detector alignment. ATLAS has performed a number of global data-taking runs in 2008 recording hundreds of thousands of tracks with hits in sub-detectors of the Inner Detector. Cosmic ray data was also recorded using pieces of the sub-detectors in a laboratory in 2006 before the inner detector was installed in the pit [13]. 


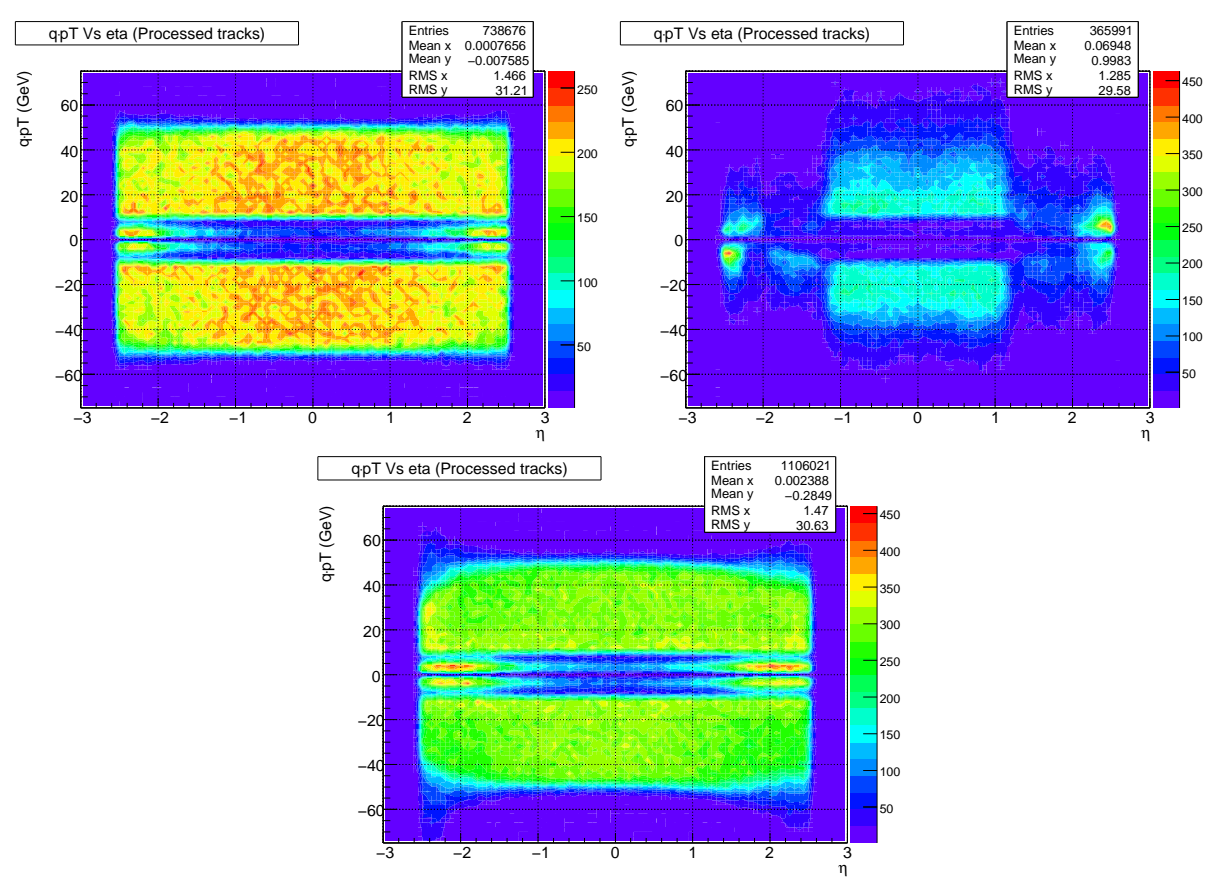

Figure 3. The $q \cdot p_{T}$ distribution for tracks as a function of the pseudorapidity. The distribution is shown using an ideally aligned detector (top left), using the expected as-built alignment precision in the FDR2 exercise (top right) and the same data from the FDR2 after calculating the first alignment constants within the 24-hours calibration loop(bottom centre).

Cosmic tracks in the full inner detector were first recorded on 14 September and alignment constants for Level 1 and 2 were calculated within a few days. Figure 4 shows the unbiased residuals for the pixel and SCT barrels. The red points show the residuals obtained when using the nominal geometry and the blue points the clear improvement when using the aligned geometry. The tracking algorithm developed for the combined test beam was used. The resolution, after alignment, is $37 \mu \mathrm{m}$ for the pixel barrel and $34 \mu \mathrm{m}$ for the SCT barrel. That this is wider than the intrinsic detector resolution is expected: all individual module degrees of freedom have not been accounted for, multiple scattering was not taken into account in the cosmic reconstruction and, because most cosmic rays arrive vertically, the side modules are not fully aligned.

Different alignment strategies yielded comparable results and within a week the new alignment constants were used for cosmic reconstruction. $\mathrm{O}(100)$ tracks were used to align the barrel at Level 1 and it is estimated that $\mathrm{O}(1$ million) would be needed to align the barrel at Level 3. Work is ongoing to more refined alignment results once sufficient data is available.

\section{Frequency scanning interferometry}

The Frequency Scanning Interferometry (FSI) $[14,15]$ optical alignment system is designed to monitor small movement of the SCT on a timescale of approximately 10 minutes. It consists of a geodetic grid capable of 842 length measurements between nodes. A laser illuminates interferometers on the geodetic grid and a reference one. Scanning the optical frequency introduces 

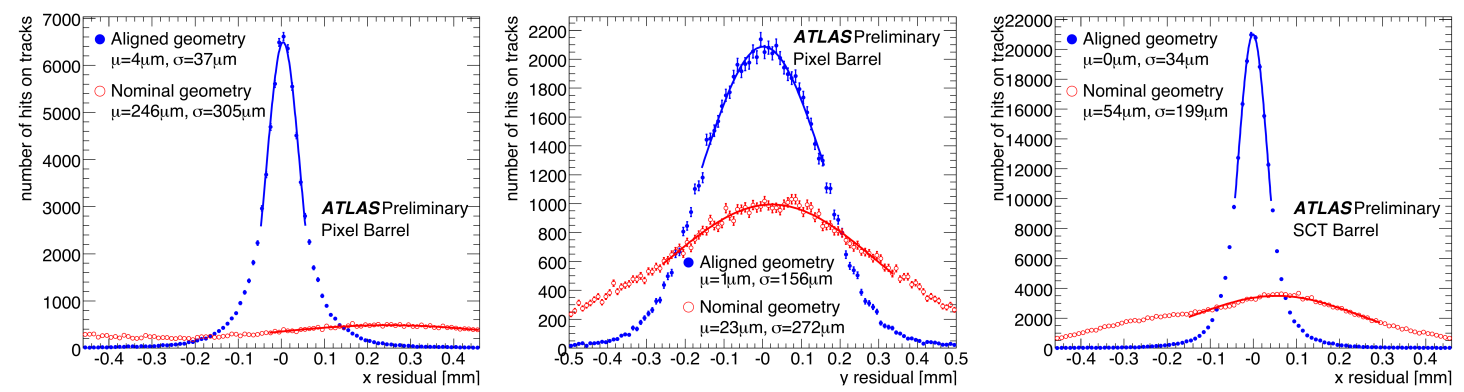

Figure 4. The unbiased residual distribution for cosmic muons in the pixel detector and the SCT using the nominal geometry (open red circles) and the aligned geometry (closed blue circles). From the left to right: the $\mathrm{X}$ residual distribution for the pixel barrel, the $\mathrm{Y}$ residual distribution for the pixel barrel and the $\mathrm{X}$ residual distribution for the SCT barrel.

a phase-shift proportional to the length of each interferometer. A comparison of the phase shift between the measured grid line and its reference determines changes in length to an accuracy of $i 1 \mu \mathrm{m}$. The system has been installed and it currently being tested. It is complementary to trackbased alignment because it monitors changes which occur on short timescales, which could include changes occurring whether the magnetic field is present or not.

\section{Conclusion}

Alignment of the ATLAS tracking detectors is a challenging task necessary for ATLAS to achieve its physics goals. Alignment from tracks produced in collisions is the primary strategy pursued and this is supported by hardware alignment and survey data. Complementary data-sets are required to constrain the weak modes.

This strategy has been tested using realistic deformation scenarios in Monte Carlo simulations. Cosmic data has recently been taken with the inner detector in its final position and first alignment results have been produced. The goal, before collisions, is to achieve full alignment at Level 2 and partial alignment at Level 3 using cosmic rays. This requires approximately 100000 tracks through the full inner detector. Using the cosmic ray data an initial set of alignment constants have been derived and therefore the ATLAS Inner Detector is ready to reconstruct the first LHC collisions.

\section{References}

[1] ATLAS collaboration, ATLAS detector and physics performance. Technical design report 2, CERN-LHCC-99-015.

[2] G. Aad et al., The ATLAS experiment at the CERN Large Hadron Collider, 2008 JINST 3 S08003.

[3] ATLAS collaboration, ATLAS inner detector: technical design report. 1, CERN-LHCC-97-016.

[4] ATLAS collaboration, ATLAS inner detector: technical design report. 2, CERN-LHCC-97-017.

[5] ATLAS collaboration, M.S. Alam et. al., ATLAS Pixel detector: technical design report, CERN-LHCC-98-013.

[6] G. Aad et al., ATLAS Pixel detector electronics and sensors, 2008 JINST 3 P07007. 
[7] ATLAS collaboration, P. Brückmann de Renstrom et al., Global $\chi^{2}$ approach to the alignment of the ATLAS silicon tracking detectors, ATL-INDET-PUB-2005-002.

[8] R. Härtel, Iterative local $\chi^{2}$ alignment approach for the ATLAS SCT detector, M.Sc. thesis, Tech. Univ. München, München, Germany (2005), MPP-2005-174.

[9] T. Göttfert, Iterative local $\chi^{2}$ alignment approach for the ATLAS Pixel detector, M.Sc. thesis, Tech. Univ. München, München, Germany (2006), MPP-2006-118.

[10] D. Hindson, A robust procedure for alignment of the ATLAS inner detector using tracks, Ph.D. thesis, Oxford Unversity, Oxford U.K. (2004).

[11] F. Heinemann, Robust track based alignment of the ATLAS silicon detectors and assessing parton distribution uncertainties in Drell-Yann processes, Ph.D. thesis, Oxford Unversity, Oxford U.K. (2004).

[12] F. Heinemann, Track based alignment of the atlas silicon detectors with the robust alignment algorithm, ATL-INDET-PUB-2007-011 [arXiv : 0710.1166].

[13] E. Abat et al., Combined performance tests before installation of the ATLAS semiconductor and transition radiation tracking detectors, 2008 JINST 3 P08003.

[14] P.A. Coe, D.F. Howell and R.B. Nickerson, Frequency scanning interferometry in ATLAS: remote, multiple, simultaneous and precise distance measurements in a hostile environment, Measur. Sci. Technol. 15 (2004) 217.

[15] S. Gibson et al., Coordinate measurement in $2 D$ and $3 D$ geometries using frequency scanning inferometry, Optics and Lasers in Engineering 43 (2005) 815. 